\title{
Posterior Interosseous Nerve Palsy Caused by a Ganglion of the Arcade of Frohse
}

\author{
Seung Jin Lee, Yoon Suk Hyun ${ }^{\varpi}$, Seung Ha Baek, Ji Hyun Seo, Hyun Ho Kim \\ Department of Orthopedic Surgery, Kangdong Sacred Heart Hospital, Hallym University Medical Center, Seoul, Korea
}

\begin{abstract}
A 51-year-old male who is right-handed visited the outpatient for right fingers-drop. The patient's fingers, including thumb, were not extended on metacarpophalangeal joint. The active motion of the right wrist was available. The electromyography and nerve conduction velocity study were consistent with the posterior interosseous neuropathy. Further evaluation was done with the magnetic resonance imaging for finding the space-occupying lesion or any possible soft tissue lesion around the radial nerve pathway. On magnetic resonance imaging, the ganglion cyst, which was about $1.8 \mathrm{~cm}$ in diameter, was observed on the proximal part of the superficial layer of the supinator muscle (Arcade of Frohse). The surgical excision was done on the base of ganglion cyst at the base of stalk of cyst which looked to be connected with proximal radioulnar joint capsule. The palsy had completely resolved when the patient was observed on the outpatient department a month after the operation.
\end{abstract}

(Clin Shoulder Elbow 2018;21(4):252-255)

Key Words: Posterior interosseous nerve palsy; Ganglions; Arcade of Frohse

Posterior interosseous nerve (PIN) palsy caused by a ganglion is uncommon and was first reported by Bowen and Stone. ${ }^{1)}$ To date, there are few reports on ganglion-induced PIN palsy. ${ }^{1-8)}$ Only one case of compressive PIN palsy caused by a ganglion has been reported in Korea. We recently experienced a similar case in Kangdong Sacred Heart Hospital and report this rare case here.

\section{Case Report}

A 51-year-old, right-handed male visited the outpatient clinic for right fingers-drop. It occurred spontaneously a month prior. The patient in this case was considered to have a brain lesion and disc disease and visited an orthopedic as an outpatient a month after seeing a neurologist. He denied any history of trauma or recent illness. During a general physical examination, the patient's fingers, including the thumb, were not extended at the metacarpophalangeal joint. Active extension of all five fingers on wrist extension revealed motor power of grade zero to one. Active motion of the right wrist was available. There was no accompanying dysesthesia or decrease of touch sensation below the elbow joint. There was no palpable mass or tenderness around the pathway of radial nerve. Plain radiographs and all routine blood studies were normal. The electromyography (EMG) and nerve conduction velocity (NCV) study were consistent with PIN. Further evaluation was performed with magnetic resonance imaging (MRI) to identify any space-occupying lesions or possible soft tissue lesions around the radial nerve pathway. On MRI imaging, a ganglion cyst about $1.8 \mathrm{~cm}$ in diameter was observed on the proximal part of the superficial layer of the supinator muscle (or Arcade of Frohse) (Fig. 1).

We proceeded with surgical removal of the cystic lesion for PIN decompression because MRI findings and the EMG-NCV study revealed definite PIN compression with a space-occupying

Received September 10, 2018. Revised October 14, 2018. Accepted October 22, 2018.

Correspondence to: Yoon Suk Hyun

Department of Orthopedic Surgery, Kangdong Sacred Heart Hospital, Hallym University Medical Center, 150 Seongan-ro, Gangdong-gu, Seoul 05355, Korea

Tel: +82-2-2224-2230, Fax: +82-2-489-4391, E-mail: yshyun72@gmail.com, ORCID: https://orcid.org/0000-0003-3826-2527

IRB approval: Kangdong Sacred Heart Hospital (No. KANGDONG 2017-10-009).

Financial support: None. Conflict of interests: None. 

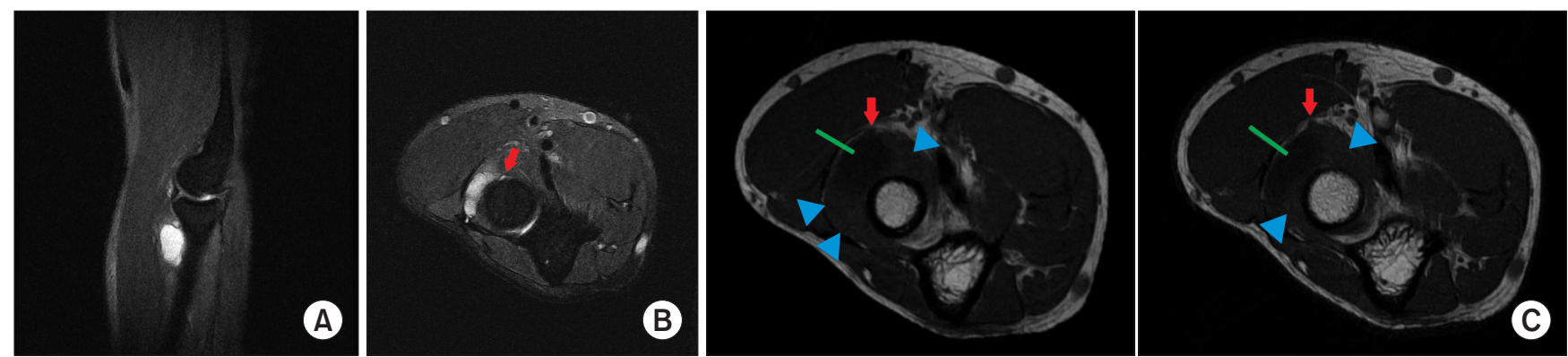

Fig. 1. (A) Sagittal T2-weighted magnetic resonance imaging (MRI) shows well-defined hyperintense cystic mass consistent with ganglion overlying the proximal radius. (B) Axial T2-weighted MRI shows well-defined hyperintense cystic mass consistent with ganglion overlying the proximal radius (arrow: ganglion stalk). (C) Two serial axial T1-weighted MRI images show posterior interosseous nerve (PIN) compression by ganglion (arrows: PIN, solid lines: ganglion cyst, arrowheads: supinator muscle).
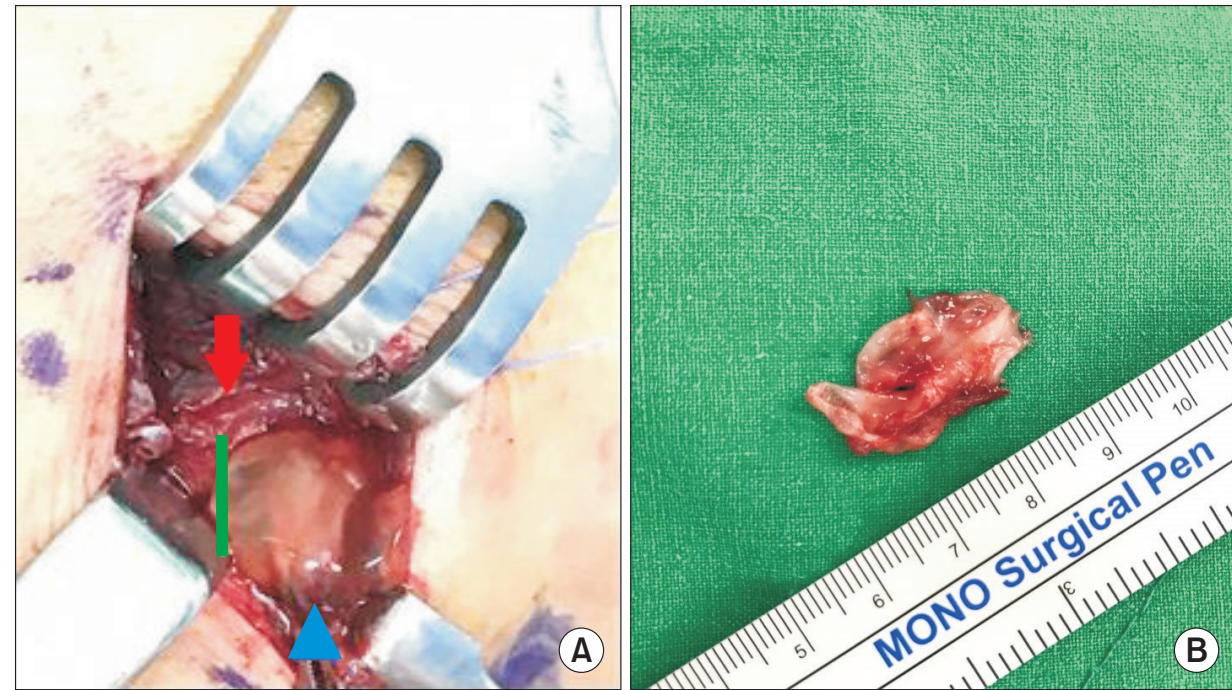

Fig. 2. (A) Brachioradialis is retracted with ragnell retractor and common extensors are retracted with Volkmann retractor $(4$ prong) (arrow: Arcade of Frohse, solid line: posterior interosseous nerve under the cyst, arrowhead: direction of stalk). (B) Ganglion cyst was excised at the base of the stalk of the cyst which seemed to be connected to the proximal radioulnar joint capsule.

lesion, although the sudden onset paralysis motor symptom had a short duration. Under general anesthesia, a modified anterolateral approach was used with a small, $4 \mathrm{~cm}$-sized incision. Instead of using the typical anterolateral approach for PIN syndrome, the cyst was reached through the space between the brachioradialis and common extensor with forearm pronation because the cyst was easily palpated just lateral to border of the brachioradialis muscle belly. The ganglion cyst was found at the proximal part of the superficial layer of the supinator muscle. Compression of the PIN by the ganglion cyst was noted. The superficial branch of the radial nerve was also visualized, and no compression was noted. Surgical excision was done at the base of the stalk of the ganglion cyst which seemed to be connected to the proximal radioulnar joint capsule (Fig. 2). The palsy had completely resolved when the patient was observed by the outpatient department a month after the operation. Patient did not complain of any motor weakness or history of recurrence during the one-year follow-up period.

\section{Discussion}

Radial nerve entrapment or compression is less common than ulnar nerve entrapment and median nerve compression in upper extremity lesion. However, the incidence rate of PIN compressive neuropathy is only $0.03 \%$ and that of superficial radial nerve compressive neuropathy is about $0.003 \%{ }^{9,10)}$ The anatomical structures that can cause radial nerve entrapment around the elbow include the fibrous band that passes over the upper arm radiocapitellar joint, the vascular leash of Henry that branches from the radial recurrent artery to the PIN, the fibrous rim of extensor carpi radialis brevis, the Arcade of Frohse, the fibrous fascia of the supinator muscle, and the thickened fascia of distal supinator muscles. ${ }^{10)}$ The most common cause of entrapment is the Arcade of Frohse. ${ }^{10)}$ The PIN is most vulnerable to compression just beyond its origin as it passes beneath the Arcade of Frohse at the proximal edge of the supinator in the radial tunnel. ${ }^{11)}$ Compression of the PIN alone may manifest as motor weakness in the distribution of the PIN, resulting in an inability to extend the metacarpophalangeal joints of the finger 
and thumb, as well as weakness in extension of the thumb at the interphalangeal joint, also called 'finger drop'. Usually there is not complete wrist drop, because the extensor carpi radialis longus and brevis are supplied by the radial nerve proximal to its terminal branch. Compression of the superficial sensory branch alone may present as pain and decreased sensation along the cutaneous area on the radial side of dorsum of the hand. ${ }^{12)}$

The identification of a compressive PIN lesion or a non-compressive PIN lesion is critical, as surgical intervention has a proven role in compressive PIN palsy but its role in non-compressive etiologies is controversial. Non-surgical management with expectant watch for 3 to 6 months is the initial treatment of choice for all forms of PIN palsy and constitutes the main treatment strategy for non-compressive PIN palsy of vasculitic and neurogenic pathologies. Surgical treatment is primarily reserved for compressive PIN palsy. The most common non-traumatic cause of compressive PIN palsy is either a bony or soft tissue growth, with the most common culprit being an inter- or intramuscular mass that is best detected by MRI scan. ${ }^{13)}$ PIN palsy caused by a ganglion is not common and was first reported by Bowen and Stone." To date, there are few reports on ganglion-induced PIN palsy. ${ }^{1-8)}$ Only one case of compressive PIN palsy caused by ganglion has been reported in Korea. Yamazaki et al. ${ }^{14)}$ reported 14 patients presenting with incomplete paralysis of the extensors of the wrist and fingers due to a ganglion at the elbow, located proximal to the Arcade of Frohse in 13 cases and distal in one case, all causing PIN palsy. Twelve patients underwent surgery and the location of the ganglion was confirmed by direct vision. One patient had puncture of the ganglion with recovery of the paralysis and therefore did not undergo surgery. The remaining patient declined surgery. All patients who underwent surgery recovered fully from the palsy within a period of 3 to 8 months after surgery. In the current case, we decided on surgical removal of the cystic lesion for PIN decompression based on the definitive PIN compression with space-occupying lesion found in the MRI and EMG-NCV study. After surgical removal, his PIN palsy was completely resolved.

In the case of PIN palsy, as in other nerve compression diseases, electrical tests are often helpful in diagnosis. Imaging tests such as ultrasound and MRI can also identify space-occupying lesions, single or multiple extensor tendon rupture, neuropathy associated with viral infection, multiple neuropathy associated with systemic diseases such as diabetes mellitus, all of which are subject to differentiation. ${ }^{15)}$

In cases where pronounced paralysis of the finger extensors is clinically observed or accompanied by a change in the PIN in the electrophysiologic examination or in the case of nerve compression due to structures such as callus, tumor or hypertrophic synovitis, surgical removal for rapid and definite improvement of $\mathrm{PIN}$ compression is preferred. ${ }^{2,16)}$

McCollam et al. ${ }^{3)}$ described three cases of PIN palsy associ- ated with a ganglion cyst arising from the superior radioulnar joint. In those cases, all three patients regained full function following surgical excision of the ganglion. The two patients with a relatively short history of PIN palsy responded quickly, with complete return of nerve function within six weeks; however, the patient in whom compressive neuropathy was present for three and half months required five months for complete recovery.

\section{References}

1. Bowen TL, Stone KH. Posterior interosseous nerve paralysis caused by a ganglion at the elbow. J Bone Joint Surg Br. 1966; 48(4):774-6.

2. Hashizume H, Nishida K, Nanba $Y$, Shigeyama $Y$, Inoue $H$, Morito $\mathrm{Y}$. Non-traumatic paralysis of the posterior interosseous nerve. J Bone Joint Surg Br. 1996;78(5):771-6.

3. McCollam SM, Corley FG, Green DP. Posterior interosseous nerve palsy caused by ganglions of the proximal radioulnar joint. J Hand Surg Am. 1988;13(5):725-8.

4. Seki Y. Posterior interosseous nerve palsy caused by a ganglion: conservative treatment with ultrasound-guided needle aspiration. J Ultrason. 2017;17(68):73-5.

5. Jou IM, Wang HN, Wang PH, Yong IS, Su WR. Compression of the radial nerve at the elbow by a ganglion: two case reports. J Med Case Rep. 2009;3:7258.

6. Tonkin MA. Posterior interosseous nerve axonotmesis from compression by a ganglion. J Hand Surg Br. 1990;15(4):491-3.

7. Mak WY. A rare cause of compression neuropathy of upper limbs - ganglionic cysts. J Orthop Trauma Rehabil. 2013;17(1): 30-2.

8. Kim YM, Kim DS, Choi ES, et al. Incomplete compressive neuropathy of posterior interosseous nerve caused by ganglion: a case report. J Korean Soc Surg Hand. 2009;14(1):28-32.

9. Latinovic R, Gulliford MC, Hughes RA. Incidence of common compressive neuropathies in primary care. J Neurol Neurosurg Psychiatry. 2006;77(2):263-5.

10. Moradi A, Ebrahimzadeh MH, Jupiter JB. Radial tunnel syndrome, diagnostic and treatment dilemma. Arch Bone Jt Surg. 2015;3(3):156-62.

11. Ogino $T$, Minami A, Kato $H$. Diagnosis of radial nerve palsy caused by ganglion with use of different imaging techniques. J Hand Surg Am. 1991;16(2):230-5.

12. Hermansdorfer JD, Greider JL, Dell PC. A case report of a compressive neuropathy of the radial sensory nerve caused by a ganglion cyst at the elbow. Orthopedics. 1986;9(7):1005-6.

13. Harshavardhana NS. Comprehensive review of isolated posterior interosseous nerve palsy. Orthop Surg Traumatol. 2017; 1(5):182-94.

14. Yamazaki $\mathrm{H}$, Kato $\mathrm{H}$, Hata $\mathrm{Y}$, Murakami N, Saitoh S. The two locations of ganglions causing radial nerve palsy. J Hand Surg Eur Vol. 2007;32(3):341-5. 
PIN Palsy Caused by a Ganglion of the Arcade of Frohse Seung Jin Lee, et al.

15. Jang HS, Lee YH. Radial nerve neuropathy. J Korean Med Assoc. 2017;60(12):958-62.

16. Cho TK, Kim JM, Bak KH, Kim CH. Posterior Interosseous
Nerve(PIN) syndrome caused by anomalous vascular leash. J Korean Neurosurg Soc. 2005;37(4):293-5. 